\title{
Functional Inequalities for Particle Systems on Polish Spaces
}

\author{
Michael Röckner ${ }^{1}$ and Feng-Yu Wang ${ }^{2 *}$ \\ ${ }^{1}$ Fakultät Für Mathematik, Universität Bielefeld, D-33501 Bielefeld, Germany \\ ${ }^{2}$ School of Mathematical Sciences, Beijing Normal University, Beijing 100875, China
}

July 1, 2018

\begin{abstract}
Various Poincaré-Sobolev type inequalities are studied for a reaction-diffusion model of particle systems on Polish spaces. The systems we consider consist of finite particles which are killed or produced at certain rates, while particles in the system move on the Polish space interacting with one another (i.e. diffusion). Thus, the corresponding Dirichlet form, which we call reaction-diffusion Dirichlet form, consists of two parts: the diffusion part induced by certain Markov processes on the product spaces $E^{n}(n \geq 1)$ which determine the motion of particles, and the reaction part induced by a $Q$-process on $\mathbb{Z}_{+}$and a sequence of reference probability measures, where the $Q$-process determines the variation of the number of particles and the reference measures describe the locations of newly produced particles. We prove that the validity of Poincaré and weak Poincaré inequalities are essentially due to the pure reaction part, i.e. either of these inequalities holds if and only if it holds for the pure reaction Dirichlet form, or equivalently, for the corresponding $Q$-process. But under a mild condition, stronger inequalities rely on both parts: the reaction-diffusion Dirichlet form satisfies a super Poincaré inequality (e.g. the log-Sobolev inequality) if and only if so do both the corresponding $Q$-process and the diffusion part. Explicit estimates of constants in the inequalities are derived. Finally, some specific examples are presented to illustrate the main results.
\end{abstract}

AMS subject Classification: 4FD0F, 60H10

Keywords: Particle system, reaction-diffusion model, Dirichlet form, functional inequality.

*Supported in part by the DFG through the Forschergruppe "Spectral Analysis, Asymptotic Distributions and Stochastic Dynamics", the BiBoS Research Centre, NNSFC(10121101), and RFDP(20040027009); Feng-Yu Wang: wangfy@bnu.edu.cn 


\section{Introduction}

In this paper we consider interacting particle systems in continnum, say in $\mathbb{R}^{d}$ or, more generally, in a Polish space $E$. At any given time we have finitely (but arbitrarily) many particles interacting with one another (called the diffusion in $E$ ). In addition, the system kills or produces particles at certain rates (called the reaction). We refer e.g. to [6] where the corresponding discretized model (on the lattice $\mathbb{Z}^{d}$ instead of $E$ ) is analyzed in detail. The main aim of this paper is to derive functional inequalities for the Dirichlet form corresponding to these systems which, as is well-known, gives information about their long-time behaviour.

Let us consider a system of finite particles on $E$ such that the number of particles behaves as a Markov chain on $\mathbb{Z}_{+}$generated by a regular $Q$-matrix $Q:=\left(q_{i j}\right)_{i, j \geq 0}$. Assume that the $Q$-process is reversible w.r.t. a probability measure $\varrho:=\left\{\varrho_{i}>0: i \geq 0\right\}$; that is,

$\left(H_{1}\right) \varrho_{i} q_{i j}=\varrho_{j} q_{j i}, \quad i, j \geq 0$.

Since we also consider the locations of particles, the state space of the underlying Markov process for the particle system is the following finite mutiple configuration space:

$$
\Gamma_{0}:=\left\{\sum_{i=1}^{n} \delta_{x_{i}}: x_{i} \in E, n \geq 0\right\}
$$

where $\delta_{x}$ is the Dirac measure at $x$ and $\sum_{i=1}^{0} \delta_{x_{i}}:=0$ is regarded as the zero measure. Let $\mathscr{F}_{\Gamma_{0}}$ be the Borel $\sigma$-field on $\Gamma_{0}$ induced by the topology of weak convergence. In particular, $\Gamma_{0}$ is metrizable to be a Polish space (cf. [1, 2, 21] and references therein for geometry and analysis on $\Gamma_{0}$ ).

To describe the reaction of the particle system, we first fix the death part of its transition rate. Since the number of particles behaves as a $Q$-process, the rate to kill $k$ particles will be $q_{|\gamma|,|\gamma|-k}$, where $\gamma \in \Gamma_{0}$ is the configuration of the system and $|\gamma|:=\gamma(E)$. Whenever the number of particles to be killed is fixed, we then simply let each particle die at the same rate. Therefore, the death part of the transition rate of the system reduces to

$$
q_{d}(\gamma, A):=\sum_{k=1}^{|\gamma|} q_{|\gamma|,|\gamma|-k} \frac{\#\left\{\eta \in \Gamma_{0}^{(k)}: \gamma-\eta \in A\right\}}{\#\left\{\eta \in \Gamma_{0}^{(k)}: \eta \leq \gamma\right\}}, \quad \Gamma_{0}^{(k)}:=\left\{\gamma \in \Gamma_{0}:|\gamma|=k\right\}, k \geq 0 .
$$

where $\#$ is the cardinality of a set and $\gamma-\eta \in A$ means that $\gamma \geq \eta$ and $\gamma-\eta \in A$. By convention we set $q_{d}(0, \cdot)=0$.

Next, we go to construct the birth part of the transition rate. Once again, since the birth rate of the particle number is determined by $Q$, we only need to fix the distributions of the newly produced particles. We shall use a sequence of measures $\left\{\mu^{(n)}\right\}$ to describe the 
distribution of new particles, where $\mu^{(n)}$ is a symmetric probability measure on $E^{n}, n \geq 1$. To this end, we need the following assumption:

$\left(H_{2}\right) \quad \mu^{(n)}$ is equivalent to $\mu^{(m)}\left(\cdot \times E^{m-n}\right)$ for any $m>n \geq 1$.

Then the birth part of the transition rate will be determined uniquely by letting the transition rate to be symmetric w.r.t. the probability measure (see Remark 2.1 below)

$$
\pi_{\mu, \varrho}(A):=\varrho_{0} 1_{B}(0)+\sum_{n=1}^{\infty} \varrho_{n} \mu^{(n)} \circ \varphi_{n}^{-1}\left(A \cap \Gamma_{0}^{(n)}\right),
$$

where

$$
\varphi_{n}(x):=\sum_{i=1}^{n} \delta_{x_{i}}, \quad n \geq 1, x:=\left(x_{1}, \cdots, x_{n}\right) \in E^{n} .
$$

It is easy to see that $\varphi_{n}$ is continuous and hence measurable.

We now describe the construction of the birth part for the transition rate. Given $m>n$, let $h_{n}^{(m)}$ be a fixed version of the density of $\mu^{(n)}$ w.r.t. $\mu^{(m)}\left(\cdot \times E^{m-n}\right)$. Let $\mu^{(m)}\left(\left(x_{1}, \cdots, x_{n}\right), \cdot\right)$ be the regular conditional distribution of $\mu^{(m)}$ given $x_{1}, \cdots, x_{n}$. If the system with $n$ particles $x_{1}, \cdots, x_{n}$ gives birth to $m-n$ new particles, then we let the distribution of the new particles be

$$
\mu_{n}^{(m)}\left(x_{1}, \cdots, x_{n} ; \cdot\right):=h_{n}^{(m)}\left(x_{1}, \cdots, x_{n}\right) \mu^{(m)}\left(\left(x_{1}, \cdots, x_{n}\right), \cdot\right) .
$$

It is trivial to see that $\mu_{n}^{(m)}$ is symmetric in $x_{1}, \cdots, x_{n}$ and

$$
\mu^{(m)}\left(\mathrm{d} x_{1}, \cdots, \mathrm{d} x_{m}\right)=\mu^{(n)}\left(\mathrm{d} x_{1}, \cdots, \mathrm{d} x_{n}\right) \mu_{n}^{(m)}\left(x_{1}, \cdots, x_{n} ; \mathrm{d} x_{n+1}, \cdots, \mathrm{d} x_{m}\right) .
$$

Since $\mu_{n}^{(m)}\left(x_{1}, \cdots, x_{n} ; A\right)$ is symmetric in $x_{1}, \cdots, x_{n}$, we may and will write

$$
\mu_{n}^{(m)}(\gamma, \cdot):=\mu_{n}^{(m)}\left(x_{1}, \cdots, x_{n} ; \cdot\right), \text { if } \gamma=\sum_{i=1}^{n} \delta_{x_{i}} .
$$

Therefore, the birth part of the transition rate can be written as follows:

$$
q_{b}(\gamma, A):=\sum_{k=1}^{\infty} q_{|\gamma|,|\gamma|+k} \mu_{|\gamma|}^{(|\gamma|+k)}\left(\gamma,\left\{x \in E^{k}: \gamma+\varphi_{k}(x) \in A\right\}\right), \quad \gamma \in \Gamma_{0}, A \in \mathscr{F}_{\Gamma_{0}} .
$$

Thus, we define the $q$-pair for the reaction of the system by letting $q(\gamma):=q\left(\gamma, \Gamma_{0}\right)=$ 
$q\left(\gamma, \Gamma_{0} \backslash\{\gamma\}\right)$ and

$$
\begin{aligned}
q(\gamma, A):= & \sum_{k=1}^{\infty} q_{|\gamma|,|\gamma|+k} \mu_{|\gamma|}^{(|\gamma|+k)}\left(\left\{x \in E^{k}: \gamma+\varphi_{k}(x) \in A\right\}\right) \\
& +\sum_{k=1}^{|\gamma|} q_{|\gamma|,|\gamma|-k} \frac{\#\left\{\eta \in \Gamma_{0}^{(k)}: \gamma-\eta \in A\right\}}{\#\left\{\eta \in \Gamma_{0}^{(k)}: \eta \leq \gamma\right\}}, \quad \gamma \in \Gamma_{0}, A \in \mathscr{F}_{\Gamma_{0}} .
\end{aligned}
$$

This $q$-pair is regular and symmetric w.r.t. $\pi_{\mu, \varrho}$ (see Propositions 2.1 and 2.2 below); that is, there exists a unique $q$-process with transition probability kernels satisfying

$$
\lim _{t \rightarrow 0} \frac{P_{t}(\gamma, A)-\delta_{\gamma}(A)}{t}=q(\gamma, A)-q(\gamma) \delta_{\gamma}(A)
$$

for all $\gamma \in \Gamma_{0}$ and $A \in \mathscr{F}_{\Gamma_{0}}$ such that $\lim _{t \rightarrow 0} \sup _{\gamma \in A}\left(1-P_{t}(\gamma,\{\gamma\})\right)=0$, and the process is reversible w.r.t. $\pi_{\mu, \varrho}$. In particular, (1.2) holds for all $A \in \mathscr{F}_{\Gamma_{0}}$ satisfying $\sup _{\gamma \in A}|\gamma|<\infty$, see e.g. [7, Theorem 1.5(1)].

Since $q(\gamma, \mathrm{d} \eta)$ is symmetric w.r.t. $\pi_{\mu, \varrho}$, the corresponding quadratic form is given by

$$
\begin{aligned}
\mathscr{E}_{R} \Gamma_{0}(F, G) & :=\frac{1}{2} \int_{\Gamma_{0} \times \Gamma_{0}}\left((F(\gamma)-F(\eta))(G(\gamma)-G(\eta)) q(\gamma, \mathrm{d} \eta) \pi_{\mu, \varrho}(\mathrm{d} \gamma)\right. \\
& =\sum_{m>n \geq 0} \int_{\Gamma_{0}^{(n)} \times \Gamma_{0}^{(m)}}(F(\gamma)-F(\eta))(G(\gamma)-G(\eta)) q_{b}(\gamma, \mathrm{d} \eta) \pi_{\mu, \varrho}(\mathrm{d} \gamma) \\
& =\sum_{n=0}^{\infty} \sum_{m=n+1}^{\infty} \varrho_{n} q_{n, m} \int_{\Gamma_{0}^{(n)}} \pi_{\mu}(\mathrm{d} \gamma) \int_{E^{m-n}}\left(D_{x} F(\gamma)\right)\left(D_{x} G(\gamma)\right) \mu_{n}^{(m)}(\gamma, \mathrm{d} x)
\end{aligned}
$$

for all $F, G$ with $\mathscr{E}_{R}^{\Gamma_{0}}(F, F)+\mathscr{E}_{R}^{\Gamma_{0}}(G, G)<\infty$, where $D_{x} F(\gamma):=F\left(\gamma+\varphi_{m-n}(x)\right)-$ $F(\gamma), x \in E^{m-n}$. To ensure that the form is well-defined in the $L^{2}\left(\Gamma_{0}, \pi_{\mu, \varrho}\right)$-sense, we assume that

$\left(H_{3}\right) \mu_{n}^{(m)}(\gamma, \cdot)$ is absolutely continuous w.r.t. $\mu^{(m-n)}$ for any $m>n \geq 0$ and any $\gamma \in \Gamma_{0}^{(n)}$.

Under this assumption $q_{b}(\gamma, \cdot)$ is absolutely continuous w.r.t. $\pi_{\mu, \varrho}$, so that $\mathscr{E}_{R} \Gamma_{0}$ is welldefined on $\mathscr{D}\left(\mathscr{E}_{R} \Gamma_{0}\right):=\left\{F \in L^{2}\left(\Gamma_{0}, \pi_{\mu, \varrho}\right): \mathscr{E}_{R} \Gamma_{0}(F, F)<\infty\right\}$; that is, $\mathscr{E}_{R} \Gamma_{0}(F, G)=$ $\mathscr{E}_{R} \Gamma_{0}\left(F^{\prime}, G^{\prime}\right)$ if $F, G$ represent the same classes as $F^{\prime}, G^{\prime}$ respectively in $L^{2}\left(\Gamma_{0}, \pi_{\mu, \varrho}\right)$. Thus, $\left(\mathscr{E}_{R} \Gamma_{0}, \mathscr{D}\left(\mathscr{E}_{R} \Gamma_{0}\right)\right)$ is a conservative symmetric Dirichlet form on $L^{2}\left(\Gamma_{0}, \pi_{\mu, \varrho}\right)$ associated to the unique reversible $q$-process (see Proposition 2.2 below).

If, in particular, $\mu^{(n)}:=\mu^{(1)^{n}}$ for all $n \geq 1$ and $q_{i, j}=0$ for $|i-j|>1$, the system is called a spatial birth-death system which goes back to [22], see also [13] for the study of a class of birth-death systems on infinite configuration spaces. In these two references the existence of the associated Markov processes and the description of reversible measures 
were studied. Recently, there has been increasing interest in the study of functional inequalities for spatial birth-death systems, see e.g. [33] for the modified log-Sobolev inequality of spatial birth-death systems on Poisson spaces, [14, 34] for the Poincaré inequality (or spectral gap) of spatial birth-death systems on configuration spaces. In this paper we first study functional inequalities for the above constructed $q$-process (i.e. the reaction process) then pass to the reaction-diffusion setting where the particles are allowed to move dependently on $E$, i.e. undergoing interactions between them.

We prove that if the support of $\mu^{(1)}$ is infinite then the Dirichlet form $\mathscr{E}_{R} \Gamma_{0}$ does not satisfy the super Poincaré inequality (hence the associated semigroup is not uniformly integrable, see [11, 29]), and it satisfies the Poincaré or the weak Poincaré inequality if and only if so does $\mathscr{E}_{Q}$, the Dirichlet form of the $Q$-matrix (see Theorem 3.1 below):

$$
\begin{aligned}
& \mathscr{E}_{Q}(\mathbf{r}, \mathbf{s}):=\sum_{n=0}^{\infty} \sum_{m=n+1}^{\infty} \varrho_{n} q_{n, m}\left(r_{n}-r_{m}\right)\left(s_{n}-s_{m}\right), \\
& \mathbf{r}=\left\{r_{n}\right\}, \mathbf{s}=\left\{s_{n}\right\} \in \mathscr{D}\left(\mathscr{E}_{Q}\right):=\left\{\mathbf{r} \in L^{2}\left(\mathbb{Z}_{+} ; \varrho\right): \mathscr{E}_{Q}(\mathbf{r}, \mathbf{r})<\infty\right\} .
\end{aligned}
$$

Furthermore, one has

$$
\operatorname{gap}\left(\mathscr{E}_{Q}\right) \geq \operatorname{gap}\left(\mathscr{E}_{R} \Gamma_{0}\right) \geq \varrho_{0} \operatorname{gap}\left(\mathscr{E}_{Q}\right),
$$

where $\operatorname{gap}(\cdot)$ is the spectral gap of a conservative Dirichlet form. The first inequality in (1.4) follows immediately by taking reference functions which are constant on each $\Gamma_{0}^{(n)}(n \geq 0)$, while to obtain the second inequality, one has to show that, up to a multiplicative constant, the Dirichlet form of a function $F$ dominates the square of the $L^{2}$ distance between $F$ and some function with constant value on each $\Gamma_{0}^{(n)}$, see the proof of Theorem 3.1 for details. Moreover, we present an example to show that in general one has $\operatorname{gap}\left(\mathscr{E}_{Q}\right)>\operatorname{gap}\left(\mathscr{E}_{R} \Gamma_{0}\right)$ (see Example 3.1 below).

Since in general $\mathscr{E}_{R} \Gamma_{0}$ does not satisfy the super Poincaré inequality, to derive stronger (e.g. the log-Sobolev) inequalities one has to enlarge the Dirichlet form. To this end, we let particles in the system move as Markov processes. More precisely, let $\left(\mathscr{E}_{0}^{(n)}, \mathscr{D}\left(\mathscr{E}_{0}^{(n)}\right)\right)$ be a symmetric conservative Dirichlet form on $L^{2}\left(E^{n} ; \mu^{(n)}\right)$. For any function $F$ on $\Gamma_{0}$, let $F^{(n)}:=F \circ \varphi_{n}$; that is,

$$
F^{(n)}\left(x_{1}, \cdots, x_{n}\right):=F\left(\sum_{i=1}^{n} \delta_{x_{i}}\right), \quad n \geq 1 .
$$

Define

$$
\mathscr{E}_{0} \Gamma_{0}(F, G):=\sum_{n=1}^{\infty} \rho_{n} \mathscr{E}_{0}^{(n)}\left(F^{(n)}, G^{(n)}\right)
$$

with $\mathscr{D}\left(\mathscr{E}_{0} \Gamma_{0}\right):=\left\{F \in L^{2}\left(\Gamma_{0}, \pi_{\mu, \varrho}\right): F^{(n)} \in \mathscr{D}\left(\mathscr{E}_{0}^{(n)}\right), n \geq 1, \mathscr{E}_{0} \Gamma_{0}(F, F)<\infty\right\}$. According to Proposition 2.3 below, $\left(\mathscr{E}_{0} \Gamma_{0}, \mathscr{D}\left(\mathscr{E}_{0} \Gamma_{0}\right)\right)$ is a conservative symmetric Dirichlet form on 
$L^{2}\left(\pi_{\mu, \varrho}\right)$. Moreover, Proposition 2.4 says that $\mathscr{E}^{\Gamma_{0}}:=\mathscr{E}_{0} \Gamma_{0}+\mathscr{E}_{R} \Gamma_{0}$ with domain $\mathscr{D}\left(\mathscr{E}^{\Gamma_{0}}\right):=$ $\mathscr{D}\left(\mathscr{E}_{0} \Gamma_{0}\right) \cap \mathscr{D}\left(\mathscr{E}_{R} \Gamma_{0}\right)$ is a symmetric Dirichlet form on $L^{2}\left(\pi_{\mu, \varrho}\right)$.

Now, we consider the $\phi$-variance inequality studied in 32] (see also [15] for a special case). This inequality interpolates the Poincaré and the log-Sobolev inequalities and has the additivity property which is in particular crucial for applications in infinite dimensions. For any probability space $(\Omega, \mathscr{B}, P)$ and any decreasing function $\phi \in C([1,2])$ with $\phi(p)>$ 0 for $p \in[1,2)$, define the $\phi$-variance by

$$
V_{\phi, P}(f):=\sup _{p \in[1,2)} \frac{P\left(f^{2}\right)-P\left(|f|^{p}\right)^{2 / p}}{\phi(p)}, \quad f \in L^{2}(P) .
$$

When $\phi \equiv 1$ and $f \geq 0$ this quantity coincides with the variance of $f$, and when $\phi(p)=$ $(2-p) / p$ it reduces to $P\left(f^{2} \log f^{2}\right)$, see e.g. [15]. Thus, the following quantity is an extension of the spectral gap and the log-Sobolev constant:

$$
\lambda_{\phi}\left(\mathscr{E}^{\Gamma_{0}}\right):=\inf \left\{\mathscr{E}^{\Gamma_{0}}(F, F): F \in \mathscr{D}\left(\mathscr{E}^{\Gamma_{0}}\right), V_{\phi, \pi_{\mu, e}}(F)=1\right\} .
$$

In particular, if $\phi \equiv 1$ then $\lambda_{\phi}=$ gap while if $\phi(p)=(2-p) / p$ then $\lambda_{\phi}\left(\mathscr{E}^{\mathscr{C}} \Gamma_{0}\right)$ coincides with the log-Sobolev constant

$$
\mathbf{L}\left(\mathscr{E}^{\Gamma_{0}}\right):=\inf \left\{\mathscr{E}^{\Gamma_{0}}(F, F): F \in \mathscr{D}\left(\mathscr{E}^{\mathscr{\Gamma} \Gamma_{0}}\right), \operatorname{Ent}_{\pi_{\mu, \varrho}}\left(F^{2}\right)=1\right\} .
$$

Let $\lambda_{\phi}\left(\mathscr{E}_{0}^{(n)}\right)$ and $\lambda_{\phi}\left(\mathscr{E}_{Q}\right)$ be the corresponding quantities of $\mathscr{E}_{0}^{(n)}\left(\right.$ w.r.t. $\left.\mu^{(n)}\right)$ and $\mathscr{E}_{Q}$ (w.r.t. @). By Theorem 4.1 below, we have

$$
\lambda_{\phi}\left(\mathscr{E}_{Q}\right) \geq \lambda_{\phi}\left(\mathscr{E}^{\Gamma_{0}}\right) \geq \lambda_{\phi}\left(\mathscr{E}_{Q}\right) \wedge \inf _{n \geq 1} \lambda_{\phi}\left(\mathscr{E}_{0}^{(n)}\right) .
$$

Moreover, let $\mathscr{E}_{0}$ be the Dirichlet form of an independent system on $L^{2}\left(E^{\mathbb{N}} ; \mu\right)$; that is, $\mu=\mu^{(1)^{\mathbb{N}}}$ and $\mathscr{E}_{0}$ is the sum of single Dirichlet forms $\left(\mathscr{E}_{0}^{(1)}, \mathscr{D}\left(\mathscr{E}_{0}^{(1)}\right)\right)$ on $L^{2}\left(E ; \mu^{(1)}\right)$. According to Theorem 4.2 below, under a mild condition, $\mathscr{E}^{\mathscr{C}} \Gamma_{0}$ satisfies the log-Sobolev (or the super log-Sobolev) inequality if and only if so do $\mathscr{E}_{0}^{(1)}$ (on $L^{2}\left(E ; \mu^{(1)}\right)$ ) and $\mathscr{E}_{Q}$ (on $\left.L^{2}\left(\mathbb{Z}_{+} ; \varrho\right)\right)$. Finally, some specific models are presented in Section 5 to illustrate the main results.

\section{The Dirichlet Forms}

Proposition 2.1. Assume $\left(H_{2}\right)$. Then $(q, q(\cdot, \mathrm{d} \gamma))$ is a totally stable and conservative $q$ pair on $\left(\Gamma_{0}, \mathscr{F}_{\Gamma_{0}}\right)$. If $\left(H_{1}\right)$ holds then $q(\cdot, \mathrm{d} \gamma)$ is symmetric w.r.t. $\pi_{\mu, \varrho}$, i.e. $\pi_{\mu, \varrho}(\mathrm{d} \gamma) q(\gamma, \mathrm{d} \eta)=$ $\pi_{\mu, \varrho}(\mathrm{d} \eta) q(\eta, \mathrm{d} \gamma)$.

Proof. Since $Q$ is totally stable, by (1.1) we have

$$
q(\gamma):=q(\gamma, E)=\sum_{k=1}^{\infty} q_{|\gamma|,|\gamma|+k}+\sum_{k=1}^{|\gamma|} q_{|\gamma|,|\gamma|-k}=q_{|\gamma|}<\infty, \quad \gamma \in \Gamma_{0},
$$


so that the $q$-pair is regular too. It remains to prove the symmetry of the measure $J(d \gamma, d \eta):=\pi_{\mu, \varrho}(\mathrm{d} \gamma) q(\gamma, d \eta)$. For any $m>n$ and measurable sets $A_{n} \subset \Gamma_{0}^{(n)}, B_{m} \subset \Gamma_{0}^{(m)}$, let $\tilde{A}_{n}:=\varphi_{n}^{-1}\left(A_{n}\right)$ and $\tilde{B}_{m}:=\varphi_{m}^{-1}\left(B_{m}\right)$. By (1.1) we have

$$
\begin{aligned}
J\left(A_{n} \times B_{m}\right) & =\int_{A_{n}} q_{b}\left(\gamma, B_{m}\right) \pi_{\mu, \varrho}(\mathrm{d} \gamma) \\
& =q_{n, m} \int_{A_{n}} \mu_{n}^{(m)}\left(y ;\left\{x \in E^{m-n}: \gamma+\varphi(x) \in B_{m}\right\}\right) \pi_{\mu, \varrho}(\mathrm{d} \gamma) \\
& =q_{n, m} \varrho_{n} \int_{\tilde{A}_{n}} \mu_{n}^{(m)}\left(y ;\left\{x \in E^{m-n}:(y, x) \in \tilde{B}_{m}\right\}\right) \mu^{(n)}(\mathrm{d} y) \\
& =q_{n, m} \varrho_{n} \mu^{(m)}\left(\left(\tilde{A}_{n} \times E^{m-n}\right) \cap \tilde{B}_{m}\right) .
\end{aligned}
$$

On the other hand,

$$
\begin{aligned}
& J\left(B_{m} \times A_{n}\right)=\int_{B_{m}} q_{d}\left(\gamma, A_{n}\right) \pi_{\mu, \varrho}(\mathrm{d} \gamma) \\
& =q_{m, n} \varrho_{m} \int_{\tilde{B}_{m}} \frac{1}{\#\left\{\eta \in \Gamma_{0}^{(n)}: \eta \leq \varphi_{m}(x)\right\}} \sum_{\eta \in \Gamma_{0}^{(n)}: \eta \leq \varphi_{m}(x)} 1_{A_{n}}(\eta) \mu^{(m)}(\mathrm{d} x) .
\end{aligned}
$$

Since $\tilde{A}_{n}, \tilde{B}_{m}$ and $\mu^{(m)}$ are symmetric in coordinates, one has

$$
\begin{aligned}
J\left(B_{m} \times A_{n}\right) & =q_{m, n} \varrho_{m} \int_{\tilde{B}_{m}} 1_{\tilde{A}_{n}}\left(x_{1}, \cdots, x_{n}\right) \mu^{(m)}\left(\mathrm{d} x_{1}, \cdots, \mathrm{d} x_{m}\right) \\
& =q_{m, n} \varrho_{m} \mu^{(m)}\left(\left(\tilde{A}_{n} \times E^{m-n}\right) \cap \tilde{B}_{m}\right) .
\end{aligned}
$$

Combining this with (2.1) and $\left(H_{1}\right)$, we obtain $J\left(A_{n} \times B_{m}\right)=J\left(B_{m} \times A_{n}\right)$. Therefore, for any measurable sets $A$ and $B$, letting $A_{n}:=A \cap \Gamma_{0}^{(n)}$ and $B_{m}:=B \cap \Gamma_{0}^{(m)}$, we have

$$
J(A \times B)=\sum_{n, m=0}^{\infty} J\left(A_{n} \times B_{m}\right)=\sum_{n, m=0}^{\infty} J\left(B_{m} \times A_{n}\right)=J(B \times A) .
$$

Remark 2.1. Once the death part $q_{d}(\gamma, \mathrm{d} \eta)$ is given, $q(\gamma, \mathrm{d} \eta)$ (hence the birth part $\left.q_{b}(\gamma, \mathrm{d} \eta)\right)$ is uniquely determined by its symmetry w.r.t. $\pi_{\mu, \varrho}$. Indeed, since the measure $J(\mathrm{~d} \gamma, \mathrm{d} \eta):=\pi_{\mu, \varrho}(\mathrm{d} \gamma) q(\gamma, \mathrm{d} \eta)$ is symmetric, it is uniquely determined by $J\left(A_{n} \times B_{m}\right)$ for $n>m$ and $A, B \in \mathscr{F}_{\Gamma_{0}}$, which depends only on $q_{d}$ and $\pi_{\mu, \varrho}$. Then $q(\gamma, \mathrm{d} \eta) / q(\gamma)$, as the regular conditional measure of the probability measure $J(\mathrm{~d} \gamma, \mathrm{d} \eta) / q(\gamma)$ given $\Gamma_{0}$, is uniquely determined by $q_{d}$ for $\pi_{\mu, \varrho^{-}}$a.e. $\Gamma_{0}$ (note that $\pi_{\mu, \varrho}(\mathrm{d} \gamma)$ is the first marginal measure of $J(\mathrm{~d} \gamma, \mathrm{d} \eta) / q(\gamma))$. 
Proposition 2.2. Assume $\left(H_{1}\right)-\left(H_{3}\right)$. Then there exists a unique q-process, which is reversible w.r.t. $\pi_{\mu, \varrho}$ with Dirichlet form $\left(\mathscr{E}_{R} \Gamma_{0}, \mathscr{D}\left(\mathscr{E}_{R} \Gamma_{0}\right)\right)$.

Proof. According to [6, Lemma 6.52], $\left(\mathscr{E}_{R}^{\Gamma_{0}}, \mathscr{D}\left(\mathscr{E}_{R} \Gamma_{0}\right)\right)$ is a Dirichlet form on $L^{2}\left(\Gamma_{0}, \pi_{\mu, \varrho}\right)$. Since $q(\gamma)=q(\gamma, E)$ by [6. Theorem 3.8], to prove the uniqueness of the $q$-process, it suffices to verify that for any bounded nonnegative measurable function $F$ such that

$$
L_{R}^{\Gamma_{0}} F(\gamma):=\int_{\Gamma_{0}}(F(\eta)-F(\gamma)) q(\gamma, \mathrm{d} \eta)=F,
$$

one has $F=0$, or equivalently, $\operatorname{dim} \mathscr{U}_{\lambda}=0$ for all $\lambda>0$ as indicated by [6, Theorem 2.37]. To this end, let $r_{0}:=F(0)$ and $r_{n}:=\frac{1}{\varrho_{n}} \int_{\Gamma_{0}^{(n)}} F \mathrm{~d} \pi_{\mu, \varrho}$ for $n \geq 1$. We first prove that $r_{n}=0$ for all $n \geq 0$. By (2.2) and the symmetry of the $q$-pair, we have

$$
\begin{aligned}
r_{n} & :=\int_{\Gamma_{0}^{(n)} \times \Gamma_{0}}(F(\eta)-F(\gamma)) \pi_{\mu, \varrho}(\mathrm{d} \gamma) q(\gamma, \mathrm{d} \eta) \\
& =\sum_{m=n+1}^{\infty} \varrho_{n} q_{n, m}\left(r_{m}-r_{n}\right)+\sum_{m=0}^{n-1} \varrho_{m} q_{m, n}\left(r_{m}-r_{n}\right) \\
& =\sum_{m=0}^{\infty} \varrho_{n} q_{n, m}\left(r_{m}-r_{n}\right) .
\end{aligned}
$$

Since the $Q$-process is unique, by this and [6. Theorem 6.42] we have $r_{n}=0$ for all $n \geq 0$, that is, $F=0 \pi_{\mu, e^{-}}$a.e. Next, it follows from $\left(H_{3}\right)$ that $q_{b}(\gamma, \cdot)$ is absolutely continuous w.r.t. $\pi_{\mu, \varrho}$. Hence (2.2) implies that

$$
\sum_{n=0}^{|\gamma|-1} \frac{q_{|\gamma|, n}}{\#\{\eta \leq \gamma:|\eta|=n\}} \sum_{\eta \leq \gamma:|\eta|=n} F(\eta)=F(\gamma)(q(\gamma)+1) .
$$

Since $\varrho_{0}>0$ and $F=0 \pi_{\mu, \varrho}$-a.e., one has $F(0)=0$ and hence by inducion in $n$ and applying the above formula, we prove that $F \equiv 0$. Thus, the $q$-process is unique and according to [6. Theorems 6.7 and 6.56], the unique $q$-process is $\pi_{\mu, \varrho}$-reversible and associated to the Dirichlet form $\left(\mathscr{E}_{R}, \mathscr{D}\left(\mathscr{E}_{R} \Gamma_{0}\right)\right)$.

Proposition 2.3. $\left(\mathscr{E}_{0} \Gamma_{0}, \mathscr{D}\left(\mathscr{E}_{0} \Gamma_{0}\right)\right)$ is a symmetric Dirichlet form on $L^{2}\left(\pi_{\mu, \varrho}\right)$.

Proof. Obviously, $\mathscr{D}\left(\mathscr{E}_{0} \Gamma_{0}\right)$ contains the set

$$
\mathscr{C}_{1}:=\left\{F \in \mathscr{C}_{0}: F^{(n)} \in \mathscr{D}\left(\mathscr{E}_{0}^{(n)}\right), n \geq 1\right\} .
$$

Since $\mathscr{D}\left(\mathscr{E}_{0}^{(n)}\right)$ is dense in $L^{2}\left(E^{n} ; \mu^{(n)}\right), \mathscr{C}_{1}$ is dense in $\mathscr{C}_{0}$ and hence in $L^{2}\left(\Gamma_{0}, \pi_{\mu, \varrho}\right)$. Next, the sub-Markovian property and the symmetry follow from that of $\mathscr{E}_{0}^{(n)}(n \geq 1)$. So, it remains to verify the closedness. Let $\left\{F_{k}\right\}_{k \geq 1}$ be a Cauchy sequence w.r.t. the corresponding Sobolev norm, and let $F$ be its limit in $L^{2}\left(\Gamma_{0}, \pi_{\mu, \varrho}\right)$. By the definition of $\mathscr{E}_{0} \Gamma_{0}$ 
one concludes that for all $m \geq 1,\left\{F_{k}^{(m)}\right\}$ is a Cauchy sequence w.r.t. the Sobolev norm induced by $\mathscr{E}_{0}$. Since $\left(\mathscr{E}_{0}, \mathscr{D}\left(\mathscr{E}_{0}\right)\right)$ is a Dirichlet form, it follows that $F^{(m)} \in \mathscr{D}\left(\mathscr{E}_{0}\right)$ and $F_{k}^{(m)} \rightarrow F^{(m)}$ in the Sobolev norm for each $m \geq 1$. Therefore, by Fatou's lemma we obtain

$$
\sum_{n=1}^{\infty} \varrho_{n} \mathscr{E}_{0}^{(n)}\left(F^{(n)}, F^{(n)}\right)=\sum_{n=1}^{\infty} \liminf _{k \rightarrow \infty} \varrho_{n} \mathscr{E}_{0}^{(n)}\left(F_{k}^{(n)}, F_{k}^{(n)}\right) \leq \liminf _{k \rightarrow \infty} \mathscr{E}_{0} \Gamma_{0}\left(F_{k}, F_{k}\right)<\infty
$$

Hence $F \in \mathscr{D}\left(\mathscr{E}_{0} \Gamma_{0}\right)$. By using Fatou's lemma again, we obtain $\mathscr{E}_{0} \Gamma_{0}\left(F-F_{k}, F-F_{k}\right) \rightarrow 0$ as $k \rightarrow \infty$.

Proposition 2.4. Let $\mathscr{D}\left(\mathscr{E}^{\Gamma_{0}}\right):=\mathscr{D}\left(\mathscr{E}_{0} \Gamma_{0}\right) \cap \mathscr{D}\left(\mathscr{E}_{R} \Gamma_{0}\right)$ and $\mathscr{E}^{\Gamma_{0}}(F, G):=\mathscr{E}_{0} \Gamma_{0}(F, G)+$ $\mathscr{E}_{R} \Gamma_{0}(F, G)$. Then $\left(\mathscr{E}^{\Gamma_{0}}, \mathscr{D}\left(\mathscr{E}^{\Gamma_{0}}\right)\right)$ is a symmetric Dirichlet form on $L^{2}\left(\pi_{\mu, \varrho}\right)$.

Proof. Simply note that $\mathscr{D}\left(\mathscr{E}^{\Gamma_{0}}\right) \supset \mathscr{C}_{1}$ and hence is dense in $L^{2}\left(\Gamma_{0}, \pi_{\mu, \varrho}\right)$. For the proof of closedness see [17, Chapter I, Section 3].

\section{Poincaré and weak Poincaré inequalities for the re- action process}

We first consider the spectral gap of $\left(\mathscr{E}_{R} \Gamma_{0}, \mathscr{D}\left(\mathscr{E}_{R} \Gamma_{0}\right)\right)$ :

$$
\operatorname{gap}\left(\mathscr{E}_{R} \Gamma_{0}\right):=\inf \left\{\mathscr{E}_{R} \Gamma_{0}(F, F): F \in \mathscr{D}\left(\mathscr{E}_{R} \Gamma_{0}\right), \pi_{\mu, \varrho}\left(F^{2}\right)=1, \pi_{\mu, \varrho}(F)=0\right\}
$$

Since $\mathscr{E}_{R} \Gamma_{0}$ is induced by the $Q$-matrix, it is natural for us to relate its spectral gap to that of $\mathscr{E}_{Q}$ :

$$
\operatorname{gap}\left(\mathscr{E}_{Q}\right):=\inf \left\{\mathscr{E}_{Q}(\mathbf{r}, \mathbf{r}): \mathbf{r} \in \mathscr{D}\left(\mathscr{E}_{Q}\right), \varrho(\mathbf{r})=0, \varrho\left(\mathbf{r}^{2}\right)=1\right\}
$$

Next, we consider the weak Poincaré inequality introduced in 23], which describes the general convergence rate of the associated semigroup:

$$
\pi_{\mu, \varrho}\left(F^{2}\right) \leq \alpha_{R}(r) \mathscr{E}_{R}^{\Gamma_{0}}(F, F)+r\|F\|_{\infty}^{2}, \quad r>0, F \in \mathscr{D}\left(\mathscr{E}_{R}^{\Gamma_{0}}\right), \pi_{\mu, \varrho}(F)=0
$$

where $\alpha_{R}:(0, \infty) \rightarrow(0, \infty)$ is a positive function. Similarly, this inequality is related to the corresponding one for $\mathscr{E}_{Q}$ :

$$
\varrho\left(\mathbf{r}^{2}\right) \leq \alpha_{Q}(r) \mathscr{E}_{Q}(\mathbf{r}, \mathbf{r})+r \sup _{n \geq 0} r_{n}^{2}, \quad r>0, \mathbf{r}=\left\{r_{n}\right\}_{n \geq 0} \in \mathscr{D}\left(\mathscr{E}_{Q}\right), \varrho(\mathbf{r})=0 .
$$

Theorem 3.1. Assume $\left(H_{1}\right)-\left(H_{3}\right)$. 
(1) $\operatorname{gap}\left(\mathscr{E}_{Q}\right) \geq \operatorname{gap}\left(\mathscr{E}_{R} \Gamma_{0}\right) \geq \varrho_{0} \operatorname{gap}\left(\mathscr{E}_{Q}\right)$. Consequently, $\operatorname{gap}\left(\mathscr{E}_{R}^{\Gamma_{0}}\right)>0$ if and only if $\operatorname{gap}\left(\mathscr{E}_{Q}\right)>0$. In particular, for the birth-death case where $l_{n}:=q_{n, n+1}>0$ for all $n \geq 0$ but $q_{n, m}=0$ for $m>n+1$, one has $\operatorname{gap}\left(\mathscr{E}_{R} \Gamma_{0}\right)>0$ if and only if

$$
\sup _{n \geq 0} \varrho([n+1, \infty)) \sum_{j=0}^{n} \frac{1}{\varrho_{j} l_{j}}<\infty
$$

(2) $\mathscr{E}_{R} \Gamma_{0}$ satisfies the weak Poincaré inequality if and only if so does $\mathscr{E}_{Q}$. More precisely, (3.1) implies (3.2) for $\alpha_{Q}=\alpha_{R}$ while (3.2) implies (3.1) for $\alpha_{R}(r)=\frac{1}{\varrho_{0}} \alpha_{Q}\left(\left(\varrho_{0} r\right) / 4\right)$.

(3) If the support of $\mu^{(1)}$ is infinite, then $\mathscr{E}_{R} \Gamma_{0}$ does not satisfy the super Poincaré inequality, i.e. the following inequality does not hold for any $\beta:(0, \infty) \rightarrow(0, \infty)$ :

$$
\pi_{\mu, \varrho}\left(F^{2}\right) \leq r \mathscr{E}_{R}^{\Gamma_{0}}(F, F)+\beta(r) \pi_{\mu, \varrho}(|F|)^{2}, \quad r>0, F \in \mathscr{D}\left(\mathscr{E}_{R} \Gamma_{0}\right) .
$$

Proof. (1) For any $\mathbf{r}=\left\{r_{n}\right\}$ with $\varrho(\mathbf{r})=0$ and $\varrho\left(\mathbf{r}^{2}\right)=1$, let $F:=\sum_{n=0}^{\infty} r_{n} 1_{\Gamma_{0}^{(n)}}$. We have $\pi_{\mu, \varrho}(F)=0$ and $\pi_{\mu, \varrho}\left(F^{2}\right)=1$, and by (1.3),

$$
\mathscr{E}_{R} \Gamma_{0}(F, F)=\sum_{n=0}^{\infty} \sum_{m=n+1}^{\infty} \varrho_{n} q_{n, m}\left(r_{n}-r_{m}\right)^{2}=\mathscr{E}_{Q}(\mathbf{r}, \mathbf{r})
$$

Then

$$
\operatorname{gap}\left(\mathscr{E}_{R}^{\Gamma_{0}}\right) \leq \inf \left\{\mathscr{E}_{Q}(\mathbf{r}, \mathbf{r}): \varrho(\mathbf{r})=0, \varrho\left(\mathbf{r}^{2}\right)=1\right\}=\operatorname{gap}\left(\mathscr{E}_{Q}\right) .
$$

Next, by the triangle inequality of the $L^{2}$-norm,

$$
\begin{aligned}
& \mathscr{E}_{R} \Gamma_{0}(F, F)=\sum_{n=0}^{\infty} \sum_{m=n+1}^{\infty} \varrho_{n} q_{n, m} \int_{E^{m}}\left(F^{(m)}(x, y)-F^{(n)}(y)\right)^{2} \mu_{n}^{(m)}(y ; \mathrm{d} x) \mu^{(n)}(\mathrm{d} y) \\
& \geq \sum_{n=0}^{\infty} \sum_{m=n+1}^{\infty} \varrho_{n} q_{n, m}\left(\sqrt{\mu^{(m)}\left(F^{(m)^{2}}\right)}-\sqrt{\mu^{(n)}\left(F^{(n)^{2}}\right)}\right)^{2} .
\end{aligned}
$$

Let

$$
\lambda_{0}\left(\mathscr{E}_{Q}\right):=\inf \left\{\mathscr{E}_{Q}(\mathbf{r}, \mathbf{r}): \varrho\left(\mathbf{r}^{2}\right)=1, r_{0}=0\right\} .
$$

It follows from (3.5) that if $F(0)=0$ then

$$
\mathscr{E}_{R} \Gamma_{0}(F, F) \geq \lambda_{0}\left(\mathscr{E}_{Q}\right) \sum_{n=0}^{\infty} \varrho_{n} \mu^{(n)}\left(F^{(n)^{2}}\right)=\lambda_{0}\left(\mathscr{E}_{Q}\right) \pi_{\mu, \varrho}\left(F^{2}\right) .
$$

Thus, for any $F \in \mathscr{D}\left(\mathscr{E}_{R} \Gamma_{0}\right)$ with $\pi_{\mu, \varrho}(F)=0$, 


$$
\mathscr{E}_{R} \Gamma_{0}(F, F)=\mathscr{E}_{R} \Gamma_{0}(F-F(0), F-F(0)) \geq \lambda_{0}\left(\mathscr{E}_{Q}\right) \pi_{\mu, \varrho}\left((F-F(0))^{2}\right) \geq \lambda_{0}\left(\mathscr{E}_{Q}\right) \pi_{\mu, \varrho}\left(F^{2}\right) .
$$

This implies that $\operatorname{gap}\left(\mathscr{E}_{R} \Gamma_{0}\right) \geq \lambda_{0}\left(\mathscr{E}_{Q}\right)$. Since for any $\mathbf{r}$ with $r_{0}=0$ and $m_{\varrho}\left(\mathbf{r}^{2}\right)=1$ one has

$$
\varrho\left(\mathbf{r}^{2}\right)-\varrho(\mathbf{r})^{2} \geq \varrho\left(\mathbf{r}^{2}\right)-\varrho\left(\mathbf{r}^{2}\right)\left(1-\varrho_{0}\right)=\varrho_{0},
$$

$\lambda_{0}\left(\mathscr{E}_{Q}\right) \geq \varrho_{0} \operatorname{gap}\left(\mathscr{E}_{Q}\right)$ and hence the desired lower bound of $\operatorname{gap}\left(\mathscr{E}_{R} \Gamma_{0}\right)$ follows. Therefore, the proof of (1) is finished by noting that for the birth-death case one has $\operatorname{gap}\left(\mathscr{E}_{Q}\right)>0$ if and only if (3.3) holds, see [20] or [7].

(2) By taking $F:=\sum_{n=0}^{\infty} r_{n} 1_{\Gamma_{0}^{(n)}}$ one concludes that (3.1) implies (3.2) for $\alpha_{Q}=\alpha_{R}$. On the other hand, for any $F \in \mathscr{D}\left(\mathscr{E}_{R} \Gamma_{0}\right)$ with $F(0)=0$, it follows from (3.5) and (3.2) that

$$
\begin{aligned}
& \alpha_{Q}(r) \mathscr{E}_{R} \Gamma_{0}(F, F) \geq \pi_{\mu, \varrho}\left(F^{2}\right)-\left(\sum_{n=1}^{\infty} \varrho_{n} \sqrt{\mu^{(n)}\left(F^{(n)^{2}}\right)}\right)^{2}-r\|F\|_{\infty}^{2} \\
& \geq \pi_{\mu, \varrho}\left(F^{2}\right) \varrho_{0}-r\|F\|_{\infty}^{2} .
\end{aligned}
$$

Therefore, for any $F \in \mathscr{D}\left(\mathscr{E}_{R} \Gamma_{0}\right)$ with $\pi_{\mu, \varrho}(F)=0$,

$$
\begin{aligned}
\pi_{\mu, \varrho}\left(F^{2}\right) & \leq \pi_{\mu, \varrho}\left((F-F(0))^{2}\right) \leq \frac{1}{\varrho_{0}} \alpha_{Q}(r) \mathscr{E}_{R} \Gamma_{0}(F, F)+\frac{r}{\varrho_{0}}\|F-F(0)\|_{\infty}^{2} \\
& \leq \frac{1}{\varrho_{0}} \alpha_{Q}(r) \mathscr{E}_{R}^{\Gamma_{0}}(F, F)+\frac{4 r}{\varrho_{0}}\|F\|_{\infty}^{2} .
\end{aligned}
$$

This implies (3.1) for $\alpha_{R}=\frac{1}{\varrho_{0}} \alpha_{Q}\left(\varrho_{0} r / 4\right)$.

(3) For any nonnegative $f \in L^{2}(\mu)$, let $F(\gamma):=\gamma(f) 1_{\Gamma_{0}^{(1)}}$. Then

$$
\pi_{\mu, \varrho}(F)=\varrho_{1} \mu^{(1)}(f), \pi_{\mu, \varrho}\left(F^{2}\right)=\varrho_{1} \mu^{(1)}\left(f^{2}\right)
$$

and

$$
\mathscr{E}_{R} \Gamma_{0}(F, F)=\varrho_{0} q_{0,1} \mu^{(1)}\left(f^{2}\right)+\varrho_{1} \sum_{m=2}^{\infty} q_{1, m} \mu^{(1)}\left(f^{2}\right) \leq\left(\varrho_{0} \vee \varrho_{1}\right) q_{1} \mu^{(1)}\left(f^{2}\right) .
$$

Thus, if the super Poincaré inequality holds then there exists a constant $c>0$ such that $\mu^{(1)}\left(f^{2}\right) \leq c \mu^{(1)}(f)^{2}$ for all nonnegative $f$, which is impossible if the support of $\mu^{(1)}$ is infinite. 
Remark 3.1. Let $\pi_{\sigma}$ be the Poisson measure with ( $\sigma$-finite) intensity $\sigma$. It is well-known that the following Poincaré inequality holds (see [33. Remark 1.4]):

$$
\pi_{\sigma}\left(F^{2}\right) \leq \int_{\Gamma_{0}} \mathrm{~d} \pi_{\sigma} \int_{E}\left(D_{x} F\right)^{2} \sigma(\mathrm{d} x)+\pi_{\sigma}(F)^{2}, \quad F \in L^{2}\left(\pi_{\sigma}\right) .
$$

See [3, 14, 34] for extensions to a class of Gibbs measures with $E=\mathbb{R}^{d}$. Thus, in our present setting one has $\operatorname{gap}\left(\mathscr{E}_{R} \Gamma_{0}\right) \geq 1$ provided $\varrho \equiv 1$ and $l_{n}:=q_{n, n+1} \geq 1, q_{n, m}=0$ for $m>n+1$. But it is easy to see that in this case (3.3) holds if and only if $\inf _{n \geq 0} n l_{n}>0$. Therefore, Theorem 3.1 (1) provides a much weaker and sharp condition for $\operatorname{gap}\left(\mathscr{E}_{R} \Gamma_{0}\right)>0$.

To conclude this section, let us present an example to show that in general $\operatorname{gap}\left(\mathscr{E}_{R} \Gamma_{0}\right)$ is strictly less than $\operatorname{gap}\left(\mathscr{E}_{Q}\right)$.

Example 3.1. Let $q_{1, k}=\beta_{k}>0$ and $q_{k, 1}=\frac{1}{2}$ for $k \neq 1$, and $q_{i, j}=0$ for $i, j \neq 1$. By $\left(H_{1}\right)$ one has $\varrho_{1}=\left(1+2 q_{1}\right)^{-1}$ and $\varrho_{k}=2 \varrho_{1} \beta_{k}(k \neq 1)$, where $q_{1}:=\sum_{k \neq 1} q_{1, k}<\infty$. Then $\operatorname{gap}\left(\mathscr{E}_{Q}\right)=\frac{1}{2}$ (see [8, Example 4.7]). On the other hand, if $\mu^{(1)}$ is non-trivial, then there exists $f \in L^{2}\left(E ; \mu^{(1)}\right)$ with $\mu^{(1)}(f)=0$ and $\mu^{(1)}\left(f^{2}\right)=1$. Let $F(\gamma):=\gamma(f) 1_{\Gamma_{0}^{(1)}}(\gamma)$. We have $\pi_{\mu, \varrho}(F)=0$ and $\pi_{\mu, \varrho}\left(F^{2}\right)=\varrho_{1}$. Moreover, by the symmetry of the $q$-pair,

$$
\mathscr{E}_{R}^{\Gamma_{0}}(F, F)=\sum_{k \neq 1} q_{k, 1} \varrho_{k}=\frac{1}{2}\left(1-\varrho_{1}\right) .
$$

Therefore,

$$
\operatorname{gap}\left(\mathscr{E}_{R} \Gamma_{0}\right) \leq \frac{1-\varrho_{1}}{2 \varrho_{1}}<\frac{1}{2}=\operatorname{gap}\left(\mathscr{E}_{Q}\right), \quad \text { if } \varrho_{1}>\frac{1}{2}
$$

\section{Functional inequalities for the reaction-diffusion process}

We first consider $\lambda_{\phi}\left(\mathscr{E}^{\Gamma_{0}}\right)$ which provides a certain exponential convergence rate of the corresponding Markov semigroup, see [32].

Theorem 4.1. Assume $\left(H_{1}\right)-\left(H_{3}\right)$. Let $\lambda_{\phi}(\cdot)$ be the quantity defined as in (1.5) for a Dirichlet form. We have

$$
\lambda_{\phi}\left(\mathscr{E}_{Q}\right) \geq \lambda_{\phi}\left(\mathscr{E}^{\Gamma_{0}}\right) \geq \lambda_{\phi}\left(\mathscr{E}_{Q}\right) \wedge \inf _{n \geq 1} \lambda_{\phi}\left(\mathscr{E}_{0}^{(n)}\right)
$$

Consequently, if $\inf _{n \geq 1} \lambda_{\phi}\left(\mathscr{E}_{0}^{(n)}\right) \geq \lambda_{\phi}\left(\mathscr{E}_{Q}\right)$, then $\lambda_{\phi}\left(\mathscr{E}^{\Gamma_{0}}\right)=\lambda_{\phi}\left(\mathscr{E}_{Q}\right)$. 
Proof. Let $F \in \mathscr{D}\left(\mathscr{E}^{\Gamma_{0}}\right)$ with $V_{\phi, \pi_{\mu, \varrho}}(F)=1$. We have

$$
\begin{aligned}
\mathscr{E}_{0} \Gamma_{0}(F, F) & :=\sum_{n=1}^{\infty} \varrho_{n} \mathscr{E}_{0}^{(n)}\left(F^{(n)}, F^{(n)}\right) \geq \inf _{n \geq 1} \lambda_{\phi}\left(\mathscr{E}_{0}^{(n)}\right) \sum_{n=1}^{\infty} \varrho_{n} V_{\phi, \pi_{\mu, \varrho}}\left(F^{(n)}\right) \\
& \geq \sup _{p \in[1,2)} \frac{\inf _{n \geq 1} \lambda_{\phi}\left(\mathscr{E}_{0}^{(n)}\right)}{\phi(p)}\left(\pi_{\mu, \varrho}\left(F^{2}\right)-\varrho_{0} F(0)^{2}-\sum_{n=1}^{\infty} \varrho_{n} \mu^{(n)}\left(\left|F^{(n)}\right|^{p}\right)^{2 / p}\right) .
\end{aligned}
$$

Next, letting $r_{0}:=F(0)$ and $r_{n}:=\mu^{(n)}\left(\left|F^{(n)}\right|^{p}\right)^{1 / p}$ for $n \geq 1$, we have

$$
\begin{aligned}
& \varrho_{0} F(0)^{2}+\sum_{n=1}^{\infty} \varrho_{n} \mu^{(n)}\left(\left|F^{(n)}\right|^{p}\right)^{2 / p}=\sum_{n=0}^{\infty} \varrho_{n} r_{n}^{2} \\
& \leq\left(\sum_{n=0}^{\infty} \varrho_{n} r_{n}^{p}\right)^{2 / p}+\frac{\phi(p) \mathscr{E}_{Q}(\mathbf{r}, \mathbf{r})}{\lambda_{\phi}\left(\mathscr{E}_{Q}\right)} \\
& =\pi_{\mu, \varrho}\left(|F|^{p}\right)^{2 / p}+\frac{\phi(p)}{\lambda_{\phi}\left(\mathscr{E}_{Q}\right)} \sum_{n=0}^{\infty} \sum_{m=n+1}^{\infty} \varrho_{n} q_{n, m}\left(r_{m}-r_{n}\right)^{2} .
\end{aligned}
$$

Since $F^{(k)}$ is symmetric, we have $\mu^{(k)}\left(\left|F^{(k)}\right|^{p}\right)=\mu^{(k)}\left(\left|F^{(k)}\right|^{p}\right)$ for any $k \geq 1$. Then, by the triangle inequality and Jensen's inequality,

$$
\begin{aligned}
\left(r_{n}-r_{m}\right)^{2} & =\left(\mu^{(n)}\left(\left|F^{(n)}\right|^{p}\right)^{1 / p}-\mu^{(m)}\left(\left|F^{(m)}\right|^{p}\right)^{1 / p}\right)^{2} \\
& \leq\left(\int_{E^{m}}\left|F^{(m)}(x, y)-F^{(n)}(y)\right|^{p} \mu_{n}^{(m)}(y ; \mathrm{d} x) \mu^{(n)}(\mathrm{d} y)\right)^{2 / p} \\
& \leq \int_{E^{m}}\left(F^{(m)}(x, y)-F^{(n)}(y)\right)^{2} \mu_{n}^{(m)}(y ; \mathrm{d} x) \mu^{(n)}(\mathrm{d} y) .
\end{aligned}
$$

Thus,

$$
\sum_{n=0}^{\infty} \sum_{m=n+1}^{\infty} \varrho_{n} q_{n, m}\left(r_{m}-r_{n}\right)^{2} \leq \mathscr{E}_{R}^{\Gamma_{0}}(F, F) .
$$

Combining this with (4.1) and (4.2), we obtain

$$
\mathscr{E}_{0} \Gamma_{0}(F, F) \geq \inf _{n \geq 1} \lambda_{\phi}\left(\mathscr{E}_{0}^{(n)}\right)\left\{V_{\phi, \pi_{\mu, \varrho}}(F)-\frac{\mathscr{E}_{R} \Gamma_{0}(F, F)}{\lambda_{\phi}\left(\mathscr{E}_{Q}\right)}\right\}
$$

Equivalently,

$$
V_{\phi, \pi_{\mu, \varrho}}(F) \leq \frac{\mathscr{E}_{0}^{\Gamma_{0}}(F, F)}{\inf _{n \geq 1} \lambda_{\phi}\left(\mathscr{E}_{0}^{(n)}\right)}+\frac{\mathscr{E}_{R}^{\Gamma_{0}}(F, F)}{\lambda_{\phi}\left(\mathscr{E}_{Q}\right)} \leq \frac{\mathscr{E}^{\Gamma_{0}}(F, F)}{\inf _{n \geq 1} \lambda_{\phi}\left(\mathscr{E}_{0}^{(n)}\right) \wedge \lambda_{\phi}\left(\mathscr{E}_{Q}\right)}
$$


This implies that $\lambda_{\phi}\left(\mathscr{E}^{\Gamma_{0}}\right) \geq \inf _{n \geq 1} \lambda_{\phi}\left(\mathscr{E}_{0}^{(n)}\right) \wedge \lambda_{\phi}\left(\mathscr{E}_{Q}\right)$. Finally, for any $\mathbf{r} \in \mathscr{D}\left(\mathscr{E}_{Q}\right)$, let $F:=\sum_{n=0}^{\infty} r_{n} 1_{\Gamma_{0}^{(n)}}$. We have $\mathscr{E}_{0} \Gamma_{0}(F, F)=0$ since $F^{(n)}:=r_{n}, n \geq 1$. Moreover, $V_{\phi, \pi_{\mu, \varrho}}(F)=$ $V_{\phi, \varrho}(\mathbf{r})$. Hence $\lambda_{\phi}\left(\mathscr{E}_{Q}\right) \geq \lambda_{\phi}\left(\mathscr{E}^{\Gamma_{0}}\right)$.

Obviously, if $\phi \in C[1,2]$ is strictly decreasing with $\phi(2)=0$ then $\lambda_{\phi}\left(\mathscr{E}^{\Gamma_{0}}\right)>0$ implies the following super Poincaré inequality for some positive function $\beta$ :

$$
\pi_{\mu, \varrho}\left(F^{2}\right) \leq r \mathscr{E}^{\Gamma_{0}}(F, F)+\beta(r) \pi_{\mu, \varrho}(|f|)^{2}, \quad r>0, F \in \mathscr{D}\left(\mathscr{E}^{\Gamma_{0}}\right) .
$$

Thus, according to Theorem [3.1, if $\mu^{(1)}$ is not finitely supported, then the non-triviality of $\mathscr{E}_{0}$ is necessary for $\lambda_{\phi}\left(\mathscr{E}^{\Gamma_{0}}\right)>0$. But in general, $\lambda_{\phi}\left(\mathscr{E}^{\Gamma_{0}}\right)>0$ only implies a certain functional inequality of $\mathscr{E}_{0}$ rather than $\inf _{n \geq 1} \lambda_{\phi}\left(\mathscr{E}_{0}^{(n)}\right)>0$. To see this, let us consider a simple situation where $\mathscr{E}_{0}$ is the Dirchlet form of an independent particle system. More precisely, let $\mu=\mu^{(1)^{\mathbb{N}}}$ be the product measure and let $\left(\mathscr{E}_{0}^{(1)}, \mathscr{D}\left(\mathscr{E}_{0}^{(1)}\right)\right)$ be a conservative symmetric Dirichlet form on $L^{2}\left(E ; \mu^{(1)}\right)$. For any $f \in L^{2}\left(E^{\mathbb{N}} ; \mu\right)$ and $x=\left(x_{1}, x_{2}, \cdots\right) \in$ $E^{\mathbb{N}}$, let

$$
f_{x \mid i}(y):=f\left(x_{1}, \cdots, x_{i-1}, y, x_{i+1}, \cdots\right), \quad i \geq 1, y \in E .
$$

Define

$$
\begin{aligned}
& \mathscr{E}_{0}(f, g):=\sum_{i=1}^{\infty} \int_{E^{\mathbb{N}}} \mathscr{E}_{0}^{(1)}\left(f_{x \mid i}, g_{x \mid i}\right) \mu(\mathrm{d} x), \\
& \mathscr{D}\left(\mathscr{E}_{0}\right):=\left\{f \in L^{2}(\mu): f_{x \mid i} \in \mathscr{D}\left(\mathscr{E}_{0}^{(1)}\right), \mu \text {-a.e. } x, i \geq 1, \mathscr{E}_{0}^{(n)}(f, f)<\infty\right\} .
\end{aligned}
$$

Then it is easy to see that $\left(\mathscr{E}_{0}, \mathscr{D}\left(\mathscr{E}_{0}\right)\right)$ is a symmetric conservative Dirichlet form on $L^{2}\left(E^{\mathbb{N}} ; \mu\right)$. Moreover, since a function on $E^{n}$ can be regarded as a cylindrical function on $E^{\mathbb{N}}$, we have the following Dirichlet forms:

$$
\mathscr{E}_{0}^{(n)}(f, g):=\mathscr{E}_{0}(f, g), \mathscr{D}\left(\mathscr{E}_{0}^{(n)}\right):=\left\{f \in L^{2}\left(E^{n} ;\left(\mu^{(1)}\right)^{n}\right): \mathscr{E}_{0}(f, f)<\infty\right\}, \quad n \geq 1 .
$$

We study the log-Sobolev inequality

$$
\pi_{\mu, \varrho}\left(F^{2} \log F^{2}\right) \leq C_{1}\left(\mathscr{E}^{\Gamma_{0}}\right) \mathscr{E}^{\Gamma_{0}}(F, F)+C_{2}\left(\mathscr{E}^{\Gamma_{0}}\right), \quad F \in \mathscr{D}\left(\mathscr{E}^{\Gamma_{0}}\right), \pi_{\mu, \varrho}\left(F^{2}\right)=1
$$

by using the following corresponding ones:

$$
\begin{gathered}
\mu^{(1)}\left(f^{2} \log f^{2}\right) \leq C_{1}\left(\mathscr{E}_{0}^{(1)}\right) \mathscr{E}_{0}^{(1)}(f, f)+C_{2}\left(\mathscr{E}_{0}^{(1)}\right), \quad f \in \mathscr{D}\left(\mathscr{E}_{0}^{(1)}\right), \mu^{(1)}\left(f^{2}\right)=1, \\
\varrho\left(\mathbf{r}^{2} \log \mathbf{r}^{2}\right) \leq C_{1}\left(\mathscr{E}_{Q}\right) \mathscr{E}_{Q}(\mathbf{r}, \mathbf{r})+C_{2}\left(\mathscr{E}_{Q}\right), \quad \mathbf{r} \in \mathscr{D}\left(\mathscr{E}_{Q}\right), \varrho\left(\mathbf{r}^{2}\right)=1 .
\end{gathered}
$$


Theorem 4.2. Assume $\left(H_{1}\right)-\left(H_{3}\right)$ and let $\mathscr{E}_{0}^{(n)}$ be given by (4.4) and (4.5).

(1) Assume that (4.7) and (4.8) hold. If there exists $\delta>0$ such that $\sum_{n=0}^{\infty} \varrho_{n} \mathrm{e}^{\delta n}<\infty$ then (4.6) holds for

$$
\begin{aligned}
& C_{1}\left(\mathscr{E}^{\Gamma_{0}}\right)=C_{1}\left(\mathscr{E}_{0}\right) \vee\left\{\left(1+\delta^{-1} C_{2}\left(\mathscr{E}_{0}\right)\right) C_{1}\left(\mathscr{E}_{Q}\right)\right\} \\
& C_{2}\left(\mathscr{E}^{\Gamma_{0}}\right)=C_{2}\left(\mathscr{E}_{Q}\right)\left(1+\delta^{-1} C_{2}\left(\mathscr{E}_{0}\right)\right)+\delta^{-1} C_{2}\left(\mathscr{E}_{0}\right) \sum_{n=0}^{\infty} \varrho_{n} \mathrm{e}^{\delta n-1} .
\end{aligned}
$$

(2) If (4.6) holds then (4.8) holds for $C_{1}\left(\mathscr{E}_{Q}\right)=C_{1}\left(\mathscr{E}^{\Gamma_{0}}\right)$ and $C_{2}\left(\mathscr{E}_{Q}\right)=C_{2}\left(\mathscr{E}^{\Gamma_{0}}\right)$, and (4.7) holds for $C_{1}\left(\mathscr{E}_{0}^{(1)}\right)=C_{1}\left(\mathscr{E}^{\Gamma_{0}}\right)$ and

$$
C_{2}\left(\mathscr{E}_{0}^{(1)}\right)=\inf _{n \geq 1} \frac{1}{n}\left\{\log \varrho_{n}+C_{1}\left(\mathscr{E}^{\Gamma_{0}}\right) q_{n}+C_{2}\left(\mathscr{E}^{\Gamma_{0}}\right)\right\}
$$

Proof. (1) Let $F \in \mathscr{D}\left(\mathscr{E}^{\Gamma_{0}}\right)$ with $\pi_{\mu, \varrho}\left(F^{2}\right)=1$. By the sub-additivity property of the entropy (see e.g. [16, (4.2)]), for any $F \in \mathscr{D}\left(\mathscr{E}_{\Gamma_{0}}\right)$ we have

$$
\operatorname{Ent}_{\mu^{(n)}}\left(F^{(n)^{2}}\right) \leq \sum_{i=1}^{n} \int_{E^{n}} \operatorname{Ent}_{\mu^{(1)}}\left(F_{x \mid i}^{(n)^{2}}\right) \mu^{(n)}(\mathrm{d} x) .
$$

Then by (4.7) we have

$$
\operatorname{Ent}_{\mu^{(n)}}\left(F^{(n)^{2}}\right) \leq C_{1}\left(\mathscr{E}_{0}\right) \mathscr{E}_{0}\left(F^{(n)}, F^{(n)}\right)+n C_{2}\left(\mathscr{E}_{0}\right) \mu^{(n)}\left(F^{(n)^{2}}\right)
$$

Thus,

$$
\begin{aligned}
\pi_{\mu, \varrho}\left(F^{2} \log F^{2}\right) \leq & \sum_{n=0}^{\infty} \varrho_{n} \mu^{(n)}\left(F^{(n)^{2}}\right) \log \mu^{(n)}\left(F^{(n)^{2}}\right) \\
& +C_{1}\left(\mathscr{E}_{0}\right) \mathscr{E}_{0} \Gamma_{0}(F, F)+C_{2}\left(\mathscr{E}_{0}\right) \sum_{n=0}^{\infty} n \varrho_{n} \mu^{(n)}\left(F^{(n)^{2}}\right) \\
\leq & \left(1+\delta^{-1} C_{2}\left(\mathscr{E}_{0}\right)\right) \sum_{n=0}^{\infty} \varrho_{n} \mu^{(n)}\left(F^{(n)^{2}}\right) \log \mu^{(n)}\left(F^{(n)^{2}}\right) \\
& +C_{1}\left(\mathscr{E}_{0}\right) \mathscr{E}_{0} \Gamma_{0}(F, F)+\delta^{-1} C_{2}\left(\mathscr{E}_{0}\right) \sum_{n=0}^{\infty} \varrho_{n} \mathrm{e}^{\delta n-1},
\end{aligned}
$$

where the last step is due to Young's inequality. Next, by (4.8) we have

$$
\begin{aligned}
& \sum_{n=0}^{\infty} \varrho_{n} \mu^{(n)}\left(F^{(n)^{2}}\right) \log \mu^{(n)}\left(F^{(n)^{2}}\right)-\pi_{\mu, \varrho}\left(F^{2}\right) \log \pi_{\mu, \varrho}\left(F^{2}\right) \\
& \leq C_{1}\left(\mathscr{E}_{Q}\right) \sum_{n=0}^{\infty} \sum_{m=n+1}^{\infty} \varrho_{n} q_{n, m}\left(\sqrt{\mu^{(n)}\left(F^{(n)^{2}}\right)}-\sqrt{\mu^{(m)}\left(F^{(m)^{2}}\right)}\right)^{2} \\
& \quad+C_{2}\left(\mathscr{E}_{Q}\right) \pi_{\mu, \varrho}\left(F^{2}\right) .
\end{aligned}
$$


We may regard $F^{(n)}$ as a function in $L^{2}\left(E^{m} ; \mu^{(m)}\right)$ so that the triangle inequality and the symmetry of $F^{(n)}$ and $F^{(m)}$ imply

$$
\begin{aligned}
\left(\sqrt{\mu^{(n)}\left(F^{(n)^{2}}\right)}-\sqrt{\mu^{(m)}\left(F^{(m)^{2}}\right)}\right)^{2} & =\left(\sqrt{\mu^{(n)}\left(F^{(n)^{2}}\right)}-\sqrt{\mu^{(m)}\left(F^{(m)^{2}}\right)}\right)^{2} \\
& \leq \mu^{(m)}\left(\left(F^{(n)}-F^{(m)}\right)^{2}\right) .
\end{aligned}
$$

Therefore, combining (4.9) and (4.10) and noting that $\pi_{\mu, \varrho}\left(F^{2}\right)=1$, we arrive at

$$
\begin{aligned}
\pi_{\mu, \varrho}\left(F^{2} \log F^{2}\right) \leq & C_{1}\left(\mathscr{E}_{0}\right) \mathscr{E}_{0}^{\Gamma_{0}}(F, F)+\left(1+\delta^{-1} C_{2}\left(\mathscr{E}_{0}\right)\right) C_{1}\left(\mathscr{E}_{Q}\right) \mathscr{E}_{R} \Gamma_{0}(F, F) \\
& +\left(1+\delta^{-1} C_{2}\left(\mathscr{E}_{0}\right)\right) C_{2}\left(\mathscr{E}_{Q}\right)+\delta^{-1} C_{2}\left(\mathscr{E}_{0}\right) \sum_{n=0}^{\infty} \varrho_{n} \mathrm{e}^{\delta n-1}
\end{aligned}
$$

(2) Assume that (4.6) holds. For any $\mathbf{r} \in \mathscr{D}\left(\mathscr{E}_{Q}\right)$, letting $F:=\sum_{n=0}^{\infty} r_{n} 1_{\Gamma_{0}^{(n)}}$ we have $\mathscr{E}_{0} \Gamma_{0}(F, F)=0$ and $\mathscr{E}_{R}^{\Gamma_{0}}(F, F)=\mathscr{E}_{Q}(\mathbf{r}, \mathbf{r})$. Moreover, $\operatorname{Ent}_{\pi_{\mu, \varrho}}\left(F^{2}\right)=\operatorname{Ent}_{\varrho}\left(\mathbf{r}^{2}\right)$ and $\pi_{\mu, \varrho}\left(F^{2}\right)=\varrho\left(\mathbf{r}^{2}\right)$. Then we obtain (4.8) for $C_{i}\left(\mathscr{E}_{Q}\right)=C_{i}\left(\mathscr{E}_{R} \Gamma_{0}\right), i=1,2$. Next, for any $f \in \mathscr{D}\left(\mathscr{E}_{0}^{(1)}\right)$ with $\mu^{(1)}\left(f^{2}\right)=1$ and any $n \geq 1$, let

$$
F(\gamma):= \begin{cases}f\left(x_{1}\right) \cdots f\left(x_{n}\right), & \text { if } \gamma=\sum_{i=1}^{n} \delta_{x_{i}} \in \Gamma_{0}^{(n)} \\ 0, & \text { otherwise }\end{cases}
$$

Then it is easy to see that

$$
\begin{aligned}
& \pi_{\mu, \varrho}\left(F^{2}\right)=\varrho_{n}, \operatorname{Ent}_{\pi_{\mu, \varrho}}\left(F^{2}\right)=n \varrho_{n} \mu^{(1)}\left(f^{2} \log f^{2}\right)-\varrho_{n} \log \varrho_{n}, \\
& \mathscr{E}_{0} \Gamma_{0}(F, F)=n \varrho_{n} \mathscr{E}_{0}^{(1)}(f, f), \quad \mathscr{E}_{R}^{\Gamma_{0}}(F, F)=\sum_{l=0}^{n-1} \varrho_{l} q_{l, n}+\sum_{l=n+1}^{\infty} \varrho_{n} q_{n, l}=\varrho_{n} q_{n},
\end{aligned}
$$

where the last equality is due to $\left(H_{1}\right)$. Thus, (4.6) implies (4.7) for the desired constants.

Now, let $\phi(p):=(2-p) / p$ so that $\lambda_{\phi}$ coincides with the log-Sobolev constant and let $\varrho$ satisfy $\sum_{n=1}^{\infty} \mathrm{e}^{\delta n} \varrho_{n}<\infty$ for some $\delta>0$. According to Theorem 4.2. if $\lambda_{\phi}\left(\mathscr{E}_{Q}\right)>0$ and (4.7) holds, then (4.6) holds. Since $\lambda_{\phi}\left(\mathscr{E}_{Q}\right)>0 \operatorname{implies} \operatorname{gap}\left(\mathscr{E}_{Q}\right)>0$, we have $\operatorname{gap}\left(\mathscr{E} \Gamma_{0}\right)>0$ according to Theorem 3.1 Thus, $\lambda_{\phi}\left(\mathscr{E}^{\Gamma_{0}}\right)>0$. On the other hand, however, there are a lot of examples where (4.7) holds but $\lambda_{\phi}\left(\mathscr{E}_{0}^{(1)}\right)=0$ (hence, $\inf _{n \geq 1} \lambda_{\phi}\left(\mathscr{E}_{0}^{(n)}\right)=0$ ). Therefore, as claimed before, $\lambda_{\phi}\left(\mathscr{E}^{\Gamma_{0}}\right)>0$ does not imply $\inf _{n \geq 1} \lambda_{\phi}\left(\mathscr{E}_{0}^{(n)}\right)>0$.

Theorem 4.2 enables us to study the super log-Sobolev inequality

$$
\pi_{\mu, \varrho}\left(F^{2} \log F^{2}\right) \leq r \mathscr{E}^{\Gamma_{0}}(F, F)+\beta(r), \quad F \in \mathscr{D}\left(\mathscr{E}^{\Gamma_{0}}\right), \pi_{\mu, \varrho}\left(F^{2}\right)=1,
$$


where $\beta:(0, \infty) \rightarrow(0, \infty)$ is a positive function. According to [9], this inequality is equivalent to the supercontractivity of $P_{t}^{\Gamma_{0}}:\left\|P_{t}^{\Gamma_{0}}\right\|_{L^{2}\left(\Gamma_{0}, \pi_{\mu, e}\right) \rightarrow L^{4}\left(\gamma, \pi_{\mu, e}\right)}<\infty$ for all $t>0$. We shall study this inequality by using the corresponding ones for $\mathscr{E}_{Q}$ and $\mathscr{E}_{0}^{(1)}$ :

$$
\begin{gathered}
\mu^{(1)}\left(f^{2} \log f^{2}\right) \leq r \mathscr{E}_{0}^{(1)}(f, f)+\beta_{0}(r), \quad f \in \mathscr{D}\left(\mathscr{E}_{0}^{(1)}\right), \mu^{(1)}\left(f^{2}\right)=1, \\
\varrho\left(\mathbf{r}^{2} \log \mathbf{r}^{2}\right) \leq r \mathscr{E}_{Q}(\mathbf{r}, \mathbf{r})+\beta_{Q}(r), \quad \mathbf{r} \in \mathscr{D}\left(\mathscr{E}_{Q}\right), \varrho\left(\mathbf{r}^{2}\right)=1 .
\end{gathered}
$$

The following result is a direct consequence of Theorem 4.2 .

Corollary 4.3. Consider the situation of Theorem 4.9 and assume that $\sum_{n=0}^{\infty} \varrho_{n} \mathrm{e}^{\delta n}<\infty$ for some $\delta>0$. Then [4.13) and [4.14) imply (4.12) for

$$
\beta(r)=\beta_{Q}\left(\delta /\left(\delta+\beta_{0}(r)\right)\right)\left(1+\delta^{-1} \beta_{0}(r)\right)+\delta^{-1} \beta_{0}(r) \sum_{n=0}^{\infty} \varrho_{n} \mathrm{e}^{\delta n-1} .
$$

On the other hand, (4.12) implies (4.14) for $\beta_{Q}=\beta$ and (4.13) for

$$
\beta_{0}(r)=\inf _{n \geq 1} \frac{1}{n}\left\{\log \varrho_{n}+\beta(r)+r q_{n}\right\} .
$$

Finally, the above arguments can be also applied to the super Poincaré inequality.

Corollary 4.4. Consider the situation of Theorem 4.2 and assume that $\operatorname{gap}\left(\mathscr{E}_{0}\right)>0$. Then $\mathscr{E}^{\Gamma_{0}}$ satisfies (4.3) for some $\beta$ if and only if there exist $\beta_{0}, \beta_{Q}:(0, \infty) \rightarrow(0, \infty)$ such that

$$
\begin{gathered}
\mu^{(1)}\left(f^{2}\right) \leq r \mathscr{E}_{0}^{(1)}(f, f)+\beta_{0}(r) \mu^{(1)}(|f|)^{2}, \quad f \in \mathscr{D}\left(\mathscr{E}_{0}^{(1)}\right), r>0, \\
\varrho\left(\mathbf{r}^{2}\right) \leq r \mathscr{E}_{Q}(\mathbf{r}, \mathbf{r})+\beta_{Q}(r) \varrho(|\mathbf{r}|)^{2}, \quad \mathbf{r} \in \mathscr{D}\left(\mathscr{E}_{Q}\right), r>0 .
\end{gathered}
$$

Proof. The proof that (4.3) implies (4.15) and (4.16) is similar to the proof that (4.6) implies (4.7) and (4.8), so we only prove the converse. Since the super Poincaré inequality is equivalent to a Sobolev type inequality, that is, replacing the function log in the $\log$-Sobolev inequality by some function increasing to infinity as the variable goes to infinity (see [11] or [29]), and since gap $\left(\mathscr{E}_{0}\right)>0$, by [32, Theorem 1.1] and (4.15) we have $\inf _{n \geq 1} \lambda_{\phi}\left(\mathscr{E}_{0}^{(n)}\right)>0$ for some strictly decreasing $\phi \in C([1,2])$ with $\phi(2)=0$. For any $F \in \mathscr{D}\left(\mathscr{E}^{\mathscr{\Gamma}} \Gamma_{0}\right)$, by the sub-additivity of $V_{\phi, \mu}$ we have

$$
V_{\phi, \mu^{(n)}}\left(F^{(n)}\right) \leq \frac{1}{\lambda_{\phi}\left(\mathscr{E}_{0}^{(1)}\right)} \mathscr{E}_{0}^{(n)}\left(F^{(n)}, F^{(n)}\right), \quad n \geq 1 .
$$


This implies

$$
\pi_{\mu, \varrho}\left(F^{2}\right) \leq \sum_{n=0}^{\infty} \varrho_{n} \mu^{(n)}\left(\left|F^{(n)}\right|^{p}\right)^{2 / p}+\frac{\phi(p)}{\lambda_{\phi}\left(\mathscr{E}_{0}^{(1)}\right)} \mathscr{E}_{0} \Gamma_{0}(F, F), \quad p \in[1,2) .
$$

Next, we claim that for any probability space $(\Omega, \mathscr{B}, P)$ and any function $h \in L^{2}(P)$ one has

$$
P\left(|h|^{p}\right)^{2 / p} \leq \frac{1}{2} P\left(h^{2}\right)+\frac{2}{p}\left(\frac{4}{p}\right)^{2 p /(2-p)} P(|h|)^{2}, \quad p \in[1,2) .
$$

Indeed, letting $P(|h|)=1$ we have

$$
P\left(|h|^{p}\right)^{2 / p} \leq \frac{2}{p}\left(P\left(|h|^{p} 1_{\{|h|>R\}}\right)^{2 / p}+R^{2}\right) \leq \frac{2}{p}\left(R^{-(2-p) / p} P\left(h^{2}\right)+R^{2}\right), \quad R>0 .
$$

Taking $R=\left(\frac{4}{p}\right)^{p /(2-p)}$ we prove (4.18). Letting $c(p):=\frac{2}{p}\left(\frac{4}{p}\right)^{2 p /(2-p)}$, by (4.17), (4.18) and (4.16) we obtain

$$
\begin{aligned}
\pi_{\mu, \varrho}\left(F^{2}\right) & \leq \frac{2 \phi(p)}{\lambda_{\phi}\left(\mathscr{E}_{0}^{(1)}\right)} \mathscr{E}_{0} \Gamma_{0}(F, F)+2 c(p) \sum_{n=0}^{\infty} \varrho_{n} \mu^{(n)}\left(\left|F^{(n)}\right|\right)^{2} \\
& \leq \frac{2 \phi(p)}{\lambda_{\phi}\left(\mathscr{E}_{0}^{(1)}\right)} \mathscr{E}_{0}^{\Gamma_{0}}(F, F)+2 c(p) r_{1} \mathscr{E}_{R}^{\Gamma_{0}}(F, F)+2 c(p) \beta_{Q}\left(r_{1}\right) \pi_{\mu, \varrho}(|F|)^{2}
\end{aligned}
$$

for any $p \in[1,2)$ and any $r_{1}>0$. Since $\phi(2)=0$, (4.3) holds with

$$
\beta(r):=\inf \left\{2 c(p) \beta_{Q}\left(r_{1}\right): p \in[1,2), r_{1}>0 \text { such that } \frac{2 \phi(p)}{\inf _{n \geq 1} \lambda_{\phi}\left(\mathscr{E}_{0}^{(n)}\right)} \vee\left(2 c(p) r_{1}\right) \leq r\right\}
$$

which is finite for any $r>0$.

\section{$5 \quad$ Examples}

In this section we present three specific models where the underlying Markov chain is the birth-death process; that is, $Q$ and $\varrho$ satisfy $\left(H_{1}\right)$ with $l_{n}:=q_{n, n+1}>0$ for $n \geq 0$ and $q_{n, m}=0$ for $m>n+1$. In the first two examples $\mathscr{E}_{0}$ refers to some infinitedimensional diffusion on a manifold, where in the first example the diffusion process is without interaction but the manifold is non-compact, and in the second example the diffusion process is given by the one-dimensional stochastic Ising model over a compact manifold. Finally, as a supplement to Theorem 3.1(3), we show in the last example that the pure reaction Dirichlet form $\mathscr{E}_{R} \Gamma_{0}$ may satisfy the log-Sobolev inequality if $E$ is finite. 
Example 5.1. (with independent diffusions) Let $M$ be connected and noncompact with Ricci curvature bounded from below. Let $V \in C(M)$ such that $V+c \rho^{\theta}$ is bounded, where $c>0, \theta>1$ are constants, and $\rho$ is the Riemannian distance function to a fixed point. By the volume comparison theorem (see [5]) one has $Z:=\int_{E} \mathrm{e}^{V(x)} \mathrm{d} x<\infty$, where $\mathrm{d} x$ is the volume measure. Let $\mu^{(1)}(\mathrm{d} x):=Z^{-1} \mathrm{e}^{V(x)} \mathrm{d} x$ and $\mathscr{E}_{0}^{(1)}(f, g):=\mu^{(1)}(\langle\nabla f, \nabla g\rangle)$ with $\mathscr{D}\left(\mathscr{E}_{0}^{(1)}\right):=H^{2,1}\left(\mu^{(1)}\right)$, the completion of $C_{0}^{\infty}(M)$ under the Sobolev norm $\|\cdot\|_{L^{2}\left(\mu^{(1)}\right)}+$ $\mathscr{E}_{0}^{(1)}(\cdot, \cdot)^{1 / 2}$. Let $\mathscr{E}_{0}$ and $\mathscr{E}_{0}^{(n)}$ be given by (4.4) and (4.5). We have:

(i) $\mathscr{E}_{R} \Gamma_{0}$ (and hence $\mathscr{E}^{\Gamma_{0}}$ ) always satisfies the weak Poincaré inequality, and it (equivalently, $\left.\mathscr{E}^{\Gamma_{0}}\right)$ satisfies the Poincaré inequality if and only if (3.3) holds.

(ii) (4.3) holds for some $\beta$ if and only if

$$
\lim _{n \rightarrow \infty} \varrho([n+1, \infty)) \sum_{j=0}^{n} \frac{1}{\varrho_{j} l_{j}}=0 .
$$

(iii) Let $\phi(p):=(2-p)^{\alpha}$ for $\alpha \in(0,1]$. Then $\lambda_{\phi}\left(\mathscr{E}^{\mathscr{\Gamma}} \Gamma_{0}\right)>0$ if and only if $\theta \geq 2 /(2-\alpha)$ and

$$
\sup _{n \geq 0} \varrho([n+1, \infty))\left(\log \varrho([n+1, \infty))^{-1}\right)^{\alpha} \sum_{j=0}^{n} \frac{1}{\varrho_{j} l_{j}}<\infty .
$$

(iv) Assume that $\sum_{n=0}^{\infty} \varrho_{n} \mathrm{e}^{\delta n}<\infty$ for some $\delta>0$. Then $\mathscr{E}^{\Gamma_{0}}$ satisfies (4.12) for some $\beta$ if and only if $\theta>2$ and

$$
\lim _{n \rightarrow \infty}\left[\varrho([n+1, \infty)) \log \varrho([n+1, \infty))^{-1}\right] \sum_{j=0}^{n} \frac{1}{\varrho_{j} l_{j}}=0 .
$$

Proof. (i) follows from Theorem 3.1 (1) and (2) and the following facts: any reversible irreducible countable Markov chain satisfies the weak Poincaré inequality (see 23. Theorem 3.1] or [31, Corollary 1.3]); gap $\left(\mathscr{E}_{0}\right)=\operatorname{gap}\left(\mathscr{E}_{0}^{(1)}\right)>0$ according to [27] or 24, Corollary 1.3]; $\operatorname{gap}\left(\mathscr{E}_{Q}\right)>0$ if and only if (3.3) holds (see [20] or [7]).

(ii) follows from Corollary 4.4 and the facts that $\operatorname{gap}\left(\mathscr{E}_{0}^{(1)}\right)>0$ and $\mathscr{E}_{0}^{(1)}$ satisfies the super Poincaré inequality since $\theta>1$ (see [28, Corollary 2.5] or [24, Corollary 1.3]), while by the discrete Hardy inequality (see [20] and [28, Theorem 4.1]), so does $\mathscr{E}_{Q}$ if and only if (5.1) holds (see also [7]).

(iv) follows from Corollary 4.3 and the facts that $\mathscr{E}_{0}^{(1)}$ satisfies the super log-Sobolev inequality if and only if $\theta>2$ (see [28, Corollaris 2.5 and 3.3]), and so does $\mathscr{E}_{Q}$ if and only if (5.3) holds (see [19]).

Finally, by [28, Corollary 2.5], (4.15) holds with $\beta_{0}(r)=\exp \left[c_{0}\left(1+r^{-1 / \alpha}\right)\right]$ for some $c_{0}>0$ if and only if $\theta \geq 2 /(2-\alpha)$. Then by [32, Corollary 1.2], $\lambda_{\phi}\left(\mathscr{E}_{0}^{(1)}\right)>0$ for the 
above $\phi$ if and only if $\theta \geq 2 /(2-\alpha)$. Therefore, (iii) follows from Theorem 4.1, Theorem 4.2 (2) and and the fact that $\lambda_{\phi}\left(\mathscr{E}_{Q}\right)>0$ if and only if (5.2) holds. The proof of this fact is similar to that presented in 18 for the $\log$-Sobolev inequality, the only difference is to use the so-called $N$-function $\Psi(r):=|r|\{\log (1+|r|)\}^{\alpha}$ in place of $|r| \log (1+|r|)$, see [26] for details.

Example 5.2. (with interacting diffusions) Let $M$ be compact and $\mathscr{J}:=\left\{J_{A}\right.$ : $A \subset \subset \mathbb{N}\}$ a smooth potential with finite range; that is, $J_{A} \in C^{\infty}\left(M^{A}\right)$ and vanishes if the diameter of $A$ is big enough. A probability measure $\mu$ on $M^{\mathbb{Z}}$ is called a Gibbs state with potential $\mathscr{J}$ if for any $A \subset \subset \mathbb{N}$, its regular conditional distribution given $x_{A^{c}} \in M^{A^{c}}$ is

$$
\mu_{A \mid x}\left(\mathrm{~d} y_{A}\right):=\frac{1}{Z_{A}\left(x_{A^{c}}\right)} \exp \left[-U_{A}\left(y_{A} \times x_{A^{c}}\right)\right] \lambda^{A}\left(\mathrm{~d} y_{A}\right),
$$

where $\lambda^{A}$ is the volume measure on $M^{A}, U_{A}:=\sum_{\Lambda: \Lambda \cap A \neq \emptyset} J_{\Lambda}$ and $Z_{A}\left(x_{A^{c}}\right)$ is the nomalization. Let $\mu^{(n)}$ be the projection of $\mu$ on $M^{\{1, \cdots, n\}}$, and let $\mathscr{E}^{(n)}$ be determined by (4.5) with

$$
\mathscr{E}_{0}(f, g):=\int_{M^{\mathbb{N}}} \sum_{k=1}^{\infty}\left\langle\nabla_{k} f, \nabla_{k} g\right\rangle \mathrm{d} \mu, \quad f, g \in \mathscr{F} C^{1}\left(M^{\mathbb{N}}\right):=\bigcup_{A \subset \subset \mathbb{N}} C^{1}\left(M^{A}\right),
$$

where $\nabla_{k}$ is the gradient w.r.t. the $k$-th component. Assume that $\mathscr{E}_{0}$ satisfies the logSobolev inequality

$$
\mu\left(f^{2} \log f^{2}\right) \leq c \mathscr{E}_{0}(f, f), \quad f \in \mathscr{F} C^{1}\left(M^{\mathbb{N}}\right), \mu\left(f^{2}\right)=1
$$

for some constant $c>0$. See e.g. [10, Theorem 2.17] for an explicit condition on $\mathscr{J}$ for (5.4) to hold. Thus, if moreover (5.2) holds for $\alpha=1$ so that $\mathscr{E}_{Q}$ satisfies the log-Sobolev inequality, then Theorem 4.2 implies the log-Sobolev for $\mathscr{E}^{\Gamma_{0}}$.

Example 5.3. (the pure reaction case with finite $E$ ) Let $E=\{1,2, \cdots, N\}$ for some $N \geq 2$ and let $\mu$ be a product probability measure on $E^{\mathbb{N}}$. Assume that $\sum_{n=1}^{\infty} \varrho_{n} \mathrm{e}^{\delta n}<$ $\infty$ for some $\delta>0$. Then $\mathscr{E}_{R} \Gamma_{0}$ satisfies the $\log$-Sobolev inequality (i.e. $\mathbf{L}\left(\mathscr{E}_{R} \Gamma_{0}\right)>0$ ) if and only if (5.2) holds with $\alpha=1$, while (4.12) holds for $\mathscr{E}_{R} \Gamma_{0}$ in place of $\mathscr{E}^{\Gamma_{0}}$ for some $\beta$ if and only if (5.3) holds. Indeed, if $E$ is finite then the trivial Dirichlet form $\mathscr{E}_{0}^{(1)}:=0$ satisfies (4.13) for some $\beta_{0}$, so that the above assertions follow from Theorem 4.2 and Corollary 4.3

Acknowledgement. The authors would like to thank Professor Mu-Fa Chen for useful suggestions and the referee for careful comments. 


\section{References}

[1] S. Albeverio, Yu. G. Kondratiev and M. Röckner, Analysis and geometry on configuration spaces, J. Funct. Anal. 154(1998), 444-500

[2] S. Albeverio, Yu. G. Kondratiev and M. Röckner, Analysis and geometry on configuration spaces: the Gibbsian case, J. Funct. Anal. 157(1998), 242-291.

[3] L. Bertini, N. Cancrini and F. Cesi, The spectral gap for a Glauber-type dynamics in a continuous gas, Ann. Inst. H. Poincaré Probab. Statist. 38(2002), 91-108.

[4] S. Bobkov and M. Ledoux, On modified logarithmic Sobolev inequalities for Bernoulli and Poisson measures, J. Funct. Anal. 156(1998), 347-365.

[5] J. Cheeger, M. Gromov and M. Taylor, Finite propagation speed, Laplace operator, and geometry of complete Riemannian manifolds, J. Diff. Geom. 17(1982), 15-53.

[6] M.-F. Chen, From Markov Chains to Non-Equilibrium Particle Systems, World Scientific, Singapore, 1992.

[7] M.-F. Chen, Ergodic convergence rates of Markov processes - eigenvalues, inequalities and ergodic theory, Proceedings of ICM (Beijing 2002), Vol. III, 41-52, Chinese High Edu. Press, Beijing 2002.

[8] M.-F. Chen and F.-Y. Wang, Cheeger's inequalities for general symmetric forms and existence criteria for spectral gap, Ann. Probab. 28(2000), 235-257.

[9] E. B. Davies and B. Simon, Ultracontractivity and the heat kernel for Schrödinger operators and Dirichlet Laplacians, J. Funct. Anal. 59(1984), 335-395.

[10] J.-D. Deuschel and D. W. Stroock, Hypercontractivity and spectral gap of symmetric diffusions with applications to the stochastic Ising models, J. Funct. Anal. 92(1990), 30-48.

[11] F.-Z. Gong and F.-Y. Wang, Functional inequalities for uniformly integrable semigroups and application to essential spectrum, Forum Math. 14(2002), 293-313.

[12] L. Gross, Logarithmic Sobolev inequalities, Amer. J. Math. 97(1976), 1061-1083.

[13] R. A. Holley and D. W. Stroock, Nearest neighbor birth and death processes on the real line, Acta Math. 140(1987), 103-154.

[14] Y. Kondratiev and E. Lytvynov, Glauber dynamics of continuous particle systems, to appear in Ann. Inst. H. Poincaré Probab. Statist.

[15] R. Latała and K. Oleszkiewicz, Between Sobolev and Poincaré, Lecture Notes Math. 1709, pp. 120-216, 1999. 
[16] M. Ledoux, On Talagrand's deviation inequalities for product measures, ESAIM: Probability and Statistics, 1(1996), 63-87.

[17] Z.-M. Ma and M. Röckner, Introduction to the Theory of (Non-Symmetric) Dirichlet Forms, Springer-Verlag, 1992.

[18] Y.-H. Mao, Logarithmic Sobolev inequalities for birth-death process and diffusion process on the line, Chinese J. Appl. Probab. Statist. 18(2002), 94-100.

[19] Y.-H. Mao, On supercontractivity for Markov semigroup, preprint.

[20] L. Miclo, An example of application of discrete Hardy's inequalities, Markov proc. Relat. Fields, 5(1999), 319-330.

[21] M. Röckner, Stochastic analysis on configuration spaces: basic ideas and recent results, In "New Directions in Dirichlet Forms", AMS/IP Stud. Adv. Math. Vol. 8, pp. 157-231, Amer. Math. Soc. Providence, RI, 1998.

[22] C. Preston, Spatial birth-and-death processes, Proceedings of the 40th Session of the International Statistical Institute (Warsaw 1975), Vol 2; Bull. Inst. Internat. Stat. 46(1975), 371-391.

[23] M. Röckner and F.-Y. Wang, Weak Poincaré inequalities and $L^{2}$-convergence rates of Markov semigroups, J. Funct. Anal. 185(2001), 564-603.

[24] M. Röckner and F.-Y. Wang, On the spectrum of a class of (nonsymmetric) diffusion operators, Bull. Lond. Math. Soc. 36(2004), 95-104.

[25] D. W. Stroock and B. Zegarlinski, The equivalence of the logarithmic Sobolev inequality and the Dobrushin-Shlosman mixing condition, Comm. Math. Phys. 144(1992), 303-323.

[26] F. Wang, Latata-Oleszkiewicz inequalities, Ph.D. Thesis, Departmnent of Mathematics, Beijing Normal University, 2003.

[27] F.-Y. Wang, Existence of spectral gap for elliptic operators, Arkiv Mat. 37(1999), 395-407.

[28] F.-Y. Wang, Functional inequalities for empty essential spectrum, J. Funct. Anal. $170(2000), 219-245$.

[29] F.-Y. Wang, Functional inequalities, semigroup properties and spectrum estimates, Infin. Dimens. Anal. Quant. Probab. Relat. Topics, 3(2000), 263-295.

[30] F.-Y. Wang, Logarithmic Sobolev inequalities: conditions and counterexamples, J. Operator Theory 46(2001), 183-197. 
[31] F.-Y. Wang, Coupling, convergence rates of Markov processes and weak Poincaré inequalities, Sci. Sin. (A) 45(2002), 975-983.

[32] F.-Y. Wang, A generalization of Poincaré and log-Sobolev inequalities, Potential Analysis 22(2005), 1-15.

[33] L. Wu. A new modified logarithmic Sobolev inequality for Point processes and several applications, Probab. Theory Relat. Fields 118(2000), 427-438.

[34] L. Wu, Estimates of spectral gap for continuous gas, Ann. Inst. H. Poincaré Probab. Statist. 40(2004), 387-409. 